\title{
Design and rationale of a randomized trial comparing the Blalock-Taussig and right ventricle-pulmonary artery shunts in the Norwood procedure
}

Richard G. Ohye, MD, ${ }^{a}$ J. William Gaynor, MD, ${ }^{b}$ Nancy S. Ghanayem, MD, ${ }^{\mathrm{c}}$ Caren S. Goldberg, MD, MS, ${ }^{a}$ Peter C. Laussen, MBBS, ${ }^{d}$ Peter C. Frommelt, MD, ${ }^{c}$ Jane W. Newburger, MD, MPH, ${ }^{d}$ Gail D. Pearson, MD, ScD, ${ }^{e}$ Sarah Tabbutt, MD, PhD, ${ }^{\mathrm{b}}$ Gil Wernovsky, MD, ${ }^{\mathrm{b}}$ Lisa M. Wruck, PhD, ${ }^{\mathrm{f}}$ Andrew M. Atz, MD, ${ }^{\mathrm{g}}$ Steve D. Colan, MD, James Jaggers, MD, ${ }^{\mathrm{h}}$ Brian W. McCrindle, MD, MPH, ${ }^{\mathrm{i}}$ Ashwin Prakash, MD, ${ }^{\mathrm{j}}$ Michael D. Puchalski, MD, ${ }^{\mathrm{k}}$ Lynn A. Sleeper, ScD, ${ }^{f}$ Mario P. Stylianou, PhD, ${ }^{e}$ and Lynn Mahony, MD, for the Pediatric Heart Network Investigators (see Appendix)

From the University of Michigan Medical School, Ann Arbor, Mich ${ }^{\mathrm{a}}$; The Children's Hospital of Philadelphia, Philadelphia, $\mathrm{Pa}^{\mathrm{b}}$; Herma Heart Center at the Children's Hospital of Wisconsin, Milwaukee, Wisconsin ${ }^{c}$; Children's Hospital Boston and Harvard Medical School, Boston, Mass ${ }^{\mathrm{d}}$; the National Heart, Lung, and Blood Institute, $\mathrm{Na}$ tional Institutes of Health, Bethesda, Md ${ }^{\text {; }}$ New England Research Institutes, Watertown, Mass ${ }^{f}$; Medical University of South Carolina, Charleston, $\mathrm{SC}^{\mathrm{g}}$; Duke University Medical Center, Durham, $\mathrm{NC}^{\text {h }}$; The Hospital for Sick Children, Toronto, Ontario, Canada' the Columbia University Medical Center, New York, NY ${ }^{j}$; Primary Children's Medical Center, Salt Lake City, Utah ${ }^{\mathrm{k}}$; and University of Texas Southwestern Medical Center, Dallas, Tex.'

Supported by U01 HL068285 (J.W.N., P.C.L.); U01 HL068270 (S.D.C., L.M., L.A.S., L.M.W.), U01 HL068269 (J.J.), U01 HL068292 (M.D.P.), U01 HL068290 (A.P.), U01 HL068288 (B.W.M.), U01 HL068281 (A.M.A.), and U01 HL068279 (W.P, S.T., G.W.) from the National Heart, Lung, and Blood Institute, National Institutes of Health, Department of Health and Human Services.

Received for publication Oct 17, 2007; accepted for publication Jan 16, 2008.

Address for reprints: Richard G. Ohye, MD, $5144 \mathrm{CVC} / \mathrm{SPC}$ 5864, 1500 East Medical Center Drive, Ann Arbor, Michigan 48109-5864 (E-mail: ohye@umich.edu).

J Thorac Cardiovasc Surg 2008;136:968-75 $0022-5223 / \$ 34.00$

Copyright $(\subset) 2008$ by The American Association for Thoracic Surgery

doi:10.1016/j.jtcvs.2008.01.013
Objective: The initial palliative procedure for patients born with hypoplastic left heart syndrome and related single right ventricle anomalies, the Norwood procedure, remains among the highest risk procedures in congenital heart surgery. The classic Norwood procedure provides pulmonary blood flow with a modified Blalock-Taussig shunt. Improved outcomes have been reported in a few small, nonrandomized studies of a modification of the Norwood procedure that uses a right ventricle-pulmonary artery shunt to provide pulmonary blood flow. Other nonrandomized studies have shown no differences between the two techniques.

Methods: The Pediatric Heart Network designed a randomized clinical trial to compare outcomes for subjects undergoing a Norwood procedure with either the right ventricle-pulmonary artery or modified Blalock-Taussig shunt. Infants with a diagnosis of single, morphologically right ventricle anomaly who are undergoing a Norwood procedure are eligible for inclusion in this study. The primary outcome is death or cardiac transplant 12 months after random assignment. Secondary outcomes include postoperative morbidity after Norwood and stage II palliation procedures, right ventricular function and pulmonary arterial growth at stage II palliation, and neurodevelopmental outcomes at 14 months old. Incidence of adverse events will also be compared between treatment groups.

Conclusion: This study will make an important contribution to the care of patients with hypoplastic left heart syndrome and related forms of single, morphologically right ventricle. It also establishes a model with which other operative interventions for patients with congenital cardiovascular malformations can be evaluated in the future.

$\mathrm{R}$ efinements in technique, perfusion management, and postoperative care have improved outcomes for patients born with hypoplastic left heart syndrome (HLHS) and other single, morphologically right ventricle (RV) conditions who undergo the Norwood procedure. Nonetheless, patients with these lesions continue to have a high mortality. ${ }^{1}$ In the classic Norwood (stage I palliation) procedure, pulmonary blood flow is provided by a modified Blalock-Taussig shunt (MBTS). A modification of this procedure involves placing a shunt from the (RV to the PA, rather than the standard MBTS, to provide pulmonary blood flow. ${ }^{2}$

Theoretic advantages of the RV-PA shunt are predominantly associated with absence of diastolic blood flow from the systemic circulation into the pulmonary circulation. They include (1) a more stable postoperative course, (2) increased coronary arterial flow because of the lack of aortic diastolic runoff, (3) improved weight gain related to improved splanchnic perfusion, and (4) lower interstage mortality. ${ }^{3-7}$ Potential disadvantages of the RV-PA shunt include the following: (1) the ventriculotomy, which could predispose toward ventricular dysfunction, arrhythmias, and false 


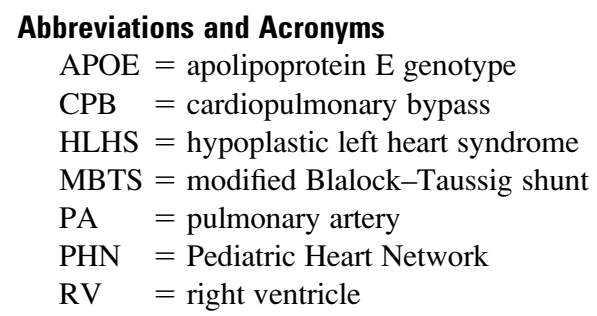

aneurysms; (2) free pulmonary insufficiency, resulting in ventricular dilation, (3) decreased PA growth related to the lack of forward flow during diastole, and (4) need for earlier stage II procedure because of hypoxemia. ${ }^{1,5,8-10}$

No prospective randomized trial has compared patient outcomes after a Norwood procedure with the MBTS with those after one incorporating the RV-PA shunt. Several case series that used historical series of patients with MBTS as a control group reported improved short-term survival with the RV-PA shunt. ${ }^{2,4,11,12}$ In contrast, a retrospective review comparing contemporaneous results of stage 1 reconstruction with the MBTS versus the RV-PA shunt performed during the same time period showed no differences in mortality or short-term outcomes. ${ }^{13}$ A second study also showed no difference in early morbidity and mortality between the two shunts, although the patients who underwent the RV-PA shunt were discharged from the hospital earlier than were those who underwent the MBTS. ${ }^{9}$ That same cohort was later reported to have no difference in mortality or morbidity after stage II palliation between MBTS and RVPA shunt groups. ${ }^{5}$ All these studies are confounded by the fact that the type of shunt placed was determined by surgeon preference. In addition, the anatomic subtypes differed significantly between cohorts. Thus there is a need for a rigorous, randomized clinical trial to determine the optimal strategy. The purpose of this report is to describe the design and rationale of a multisite, randomized clinical trial to compare outcomes associated with the RV-PA shunt with those of the MBTS in a prospective and randomized fashion.

\section{Materials and Methods Study Overview}

This trial will test the hypothesis that the RV-PA shunt is associated with a decrease relative to the MBTS in the frequency of the combined end point of mortality or transplant at 12 months after random assignment. We plan to enroll 466 infants to be randomly assigned to undergo either MBTS or RV-PA shunt. The concept for this study was developed by investigators at the University of Michigan Medical School, the Children's Hospital of Philadelphia, Children's Hospital of Wisconsin, and Children's Hospital Boston, who collaborated with the National Heart, Lung, and Blood Institute-funded Pediatric Heart Network (PHN) ${ }^{14}$ to develop the final study design. The study is being conducted at 14 clinical centers. A flow chart of

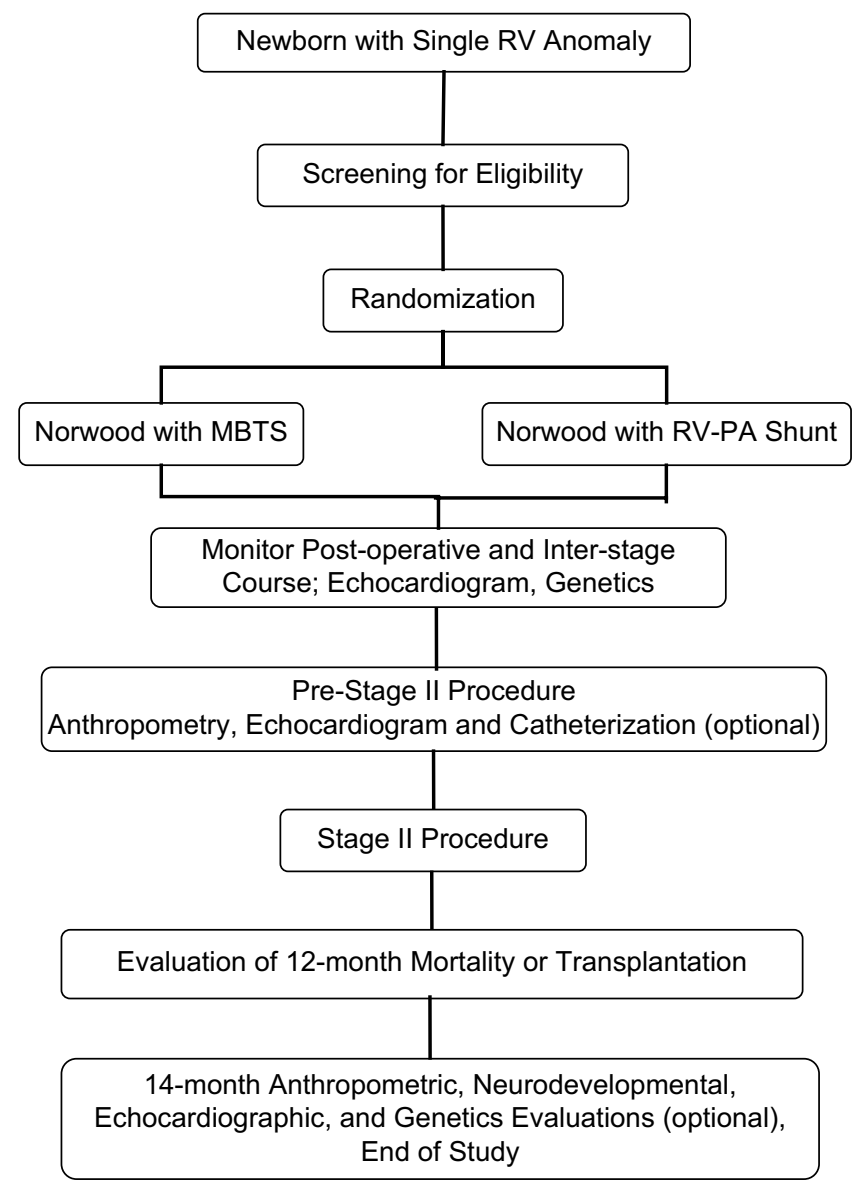

Figure 1. Flow diagram for trial. $R V$, Right ventricle; MBTS, modified Blalock-Taussig shunt; $P A$, pulmonary artery.

the study design is shown in Figure 1. An independent Data and Safety Monitoring Board and an independent medical monitor have been established by the National Heart, Lung, and Blood Institute to monitor this trial for data quality and safety.

\section{Subject Selection}

Subject selection criteria were defined to ensure a relatively homogeneous group of subjects with a functionally single ventricle lesion characterized by a single morphologically RV. Inclusion and exclusion criteria are shown in Table 1.

\section{Randomization and Stratification}

Subjects are assigned to treatment group with randomly permuted blocks within strata defined by the presence or absence of aortic atresia and the presence or absence of obstructed pulmonary venous return. ${ }^{15-18}$ Obstructed pulmonary venous return is defined by the use of postnatal intervention, including balloon septostomy, open atrial septectomy, or urgent Norwood procedure. Aortic atresia is defined by 2-dimensional and color Doppler echocardiographic findings interpreted at the local center. Because of potential differences in surgeon experience and operative techniques, dynamic balancing by surgeon ensures that treatment arm totals are balanced within each 


\section{TABLE 1. Inclusion and exclusion criteria}

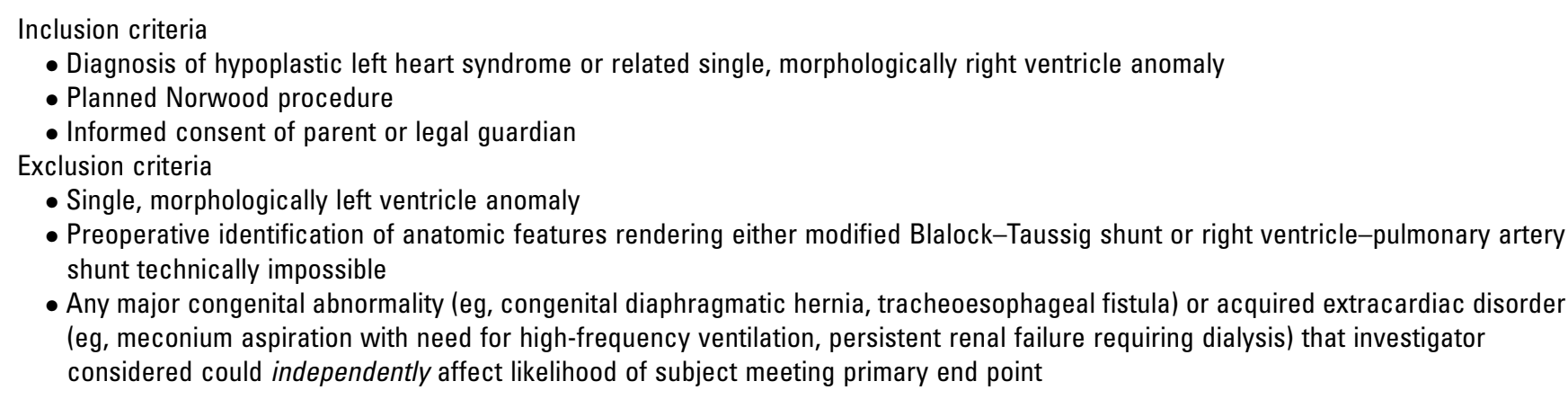

surgeon's case load and will avoid excessively small stratum sizes that might result from use of more than two stratification factors.

\section{Technical Considerations of Surgery}

Each center will strive to maintain a consistent approach to perioperative care and the conduct of the operation, regardless of the shunt group to which the subject is randomly assigned, for the duration of the trial. These variables include such techniques as cardiopulmonary bypass $(\mathrm{CPB}$, including the use of regional cerebral perfusion, hematocrit levels, and methods of $\mathrm{pH}$ monitoring) and management in the intensive care unit before and after surgery. Despite these efforts to standardize the care for the duration of the trial, however, management techniques invariably change with time. To minimize the impact of these changes, permuted block randomization will be used, as mentioned previously. In addition, extensive data on perioperative management will be recorded to allow analysis of significant changes in management during the trial time frame.

The MBTS and RV-PA shunts are constructed of unvalved polytetrafluoroethylene tube grafts with standard surgical techniques. ${ }^{2,16}$ Features of cardiac anatomy recognized in the operating room after random assignment occasionally render the performance of the MBTS or RV-PA shunt technically infeasible. Although every effort is made to use the shunt to which the subject has been randomly assigned, the surgeon may choose an alternative shunt at any time during the operation if it is deemed to be in the subject's best interest because of unanticipated anatomic constraints. If the innominate artery is not appropriate for the MBTS, such as in the scenario of an aberrant right subclavian artery and inadequate caliber right carotid artery, the subject will be considered for an aortopulmonary shunt. Because a shunt arising from the aorta is a shunt from a systemic artery to the PA, its use will not be considered a treatment crossover. On rare occasions, if the surgeon considers that the MBTS or another shunt from a systemic artery to the PA cannot be performed, a crossover will occur. Another possibility is the finding of a large conal branch on the RV free wall in the area of the proposed origin of the RV-PA shunt. In this case, the subject will also cross over to the alternative shunt. It is also possible that after the placement of the initial randomly assigned shunt, the subject may not be able to be separated successfully from CPB. The surgeon may place another type of shunt in an attempt to save the subject's life.

\section{Study Measurements and Subject Follow-up}

Baseline data. Demographic and preoperative data include pregnancy history, fetal diagnosis, birth weight, race, gender, gesta- tional age, Apgar scores, clinical status and anatomic diagnosis at presentation, occurrence of important preoperative complications, age at operation, and genetic evaluation findings (if performed). All centers perform 2-dimensional echocardiographic studies, which are reviewed both locally and in the 2-dimensional echocardiography core laboratory to evaluate anatomy and ventricular function. Centers with 3-dimensional echocardiography capability also collect these studies for local interpretation and review by the 3dimensional echocardiography core laboratory. Any additional interventions and important events, as defined by the protocol, are recorded.

Follow-up visits. The schedule of data collection is summarized in Table 2. Follow-up data are obtained at five times: (1) during hospitalization for the Norwood procedure, (2) before the stage II palliation, (3) during hospitalization for the stage II palliation, (4) 12 months after random assignment, and (5) at age 14 months. Specific data and studies include the following:

- echocardiographic and Doppler images obtained for analysis after the Norwood procedure, before the stage II palliation and at age 14 months;

- height, weight, and head circumference measured at the time of each echocardiogram;

- apolipoprotein E genotype (APOE) obtained by buccal swab from subjects with consent during the hospitalization for the Norwood procedure (consent for genotyping not required for participation in the main trial);

- Cardiac catheterization data and images, if a routine cardiac catheterization is performed for clinical indications before the stage II palliation;

- Vital status and whether a heart transplant has been performed (collected 12 months after random assignment only).

- The Bayley Scales of Infant Development-II (Harcourt Assessment, Inc, San Antonio, Tex), MacArthur-Bates Communicative Development Inventories (Singular Publishing Group, San Diego, Calif), Hollingshead Four Factor Scale (Four-factor index of social status [unpublished manuscript]; Yale University, New Haven, Conn), and Functional Status II-Revised questionnaire (Albert Einstein College of Medicine, Bronx, NY) at 14 months old.

Echocardiographic data, APOE genotype, and cardiac catheterization data are centrally interpreted in PHN core laboratories. To cover long-term follow-up of this unique patient population, the consent form includes permission to contact each family annually until the subject reaches the age of 5 years. 
TABLE 2. Schedule of trial measurements

\begin{tabular}{|c|c|c|c|c|c|c|c|}
\hline \multirow[b]{2}{*}{ Measurement } & \multirow{2}{*}{$\frac{\text { Baseline }}{\text { Study entry }}$} & \multicolumn{6}{|c|}{ Follow-up } \\
\hline & & Norwood hosp & Discharge & Before stage II & Stage II hosp & Age $12 \mathrm{mo}$ & Age $14 \mathrm{mo}$ \\
\hline Medical history & $\mathrm{X}$ & & $x$ & $\mathrm{X}$ & & & $\mathrm{X}$ \\
\hline Height, weight, and head circumference & $\mathrm{X}$ & & $X$ & $\mathrm{X}$ & & & $X$ \\
\hline Anatomic subtype & $\mathrm{X}$ & & & & & & \\
\hline Intensive care unit and hospital courses & & $X$ & & & $X$ & & \\
\hline Echocardiogram & $X$ & & $x$ & $\mathrm{X}$ & & & $\mathrm{X}$ \\
\hline Catheterization* & & & & $\mathrm{X}$ & & & \\
\hline Apolipoprotein E genotype $\dagger$ & & $X$ & & & & & \\
\hline Death or transplant & & & & & & $x$ & \\
\hline Neurodevelopmental evaluation & & & & & & & $\mathrm{X}$ \\
\hline Clinical genetics evaluation $\dagger$ & & $x$ & & & & & $\mathrm{X}$ \\
\hline
\end{tabular}

hosp, Hospitalization. * Not mandated by protocol, performed at discretion of attending cardiologist. †Not mandated by protocol, performed only with consent of family.

\section{Trial Outcomes}

The primary outcome is death or cardiac transplant by 12 months after randomization. Secondary outcomes are listed in Table 3.

\section{Blinding of Treatment Group Assignment}

This trial is not blinded with respect to knowledge of treatment assignment. Blinding is not feasible for most of the personnel caring for the subjects, because the treatment strategies produce differences in physical examination findings and in the follow-up studies performed for the secondary end points, such as echocardiography and cardiac catheterization. Only the specialists who perform the neurodevelopmental testing and the APOE genotyping are blinded to treatment assignment.

\section{Statistical Considerations}

For the primary trial result, the proportion of subjects with MBTS versus RV-PA shunt reaching the primary trial end point by 12 months after randomization will be compared with a Fisher exact test. Furthermore, to test the hypothesis that differences in the surgical strategies are present early, a comparison of the primary end point in the two trial arms will be conducted with the Kaplan-Meier method for estimation and the Gehan-Wilcoxon test, which places more weight on early failure times because of weights determined by risk set sample size. A competing risks analysis (death vs transplant vs stage II surgery performed vs no progression through additional stages) will also be conducted to characterize subject risk more fully by treatment group.

All primary analyses will be performed on an intention-to-treat basis. This approach implies that the rare randomly allocated subject who receives neither trial shunt (eg, in the event of death between assignment and the Norwood procedure) would remain classified in the assigned surgical group for analysis. If it is clear that the decision not to proceed with the Norwood procedure has not been influenced by knowledge of the assigned shunt, however, then no bias is incurred, intention-to-treat is preserved, and those subjects will be excluded from analysis. A review panel will evaluate each case in which a randomly allocated subject receives neither trial shunt. If the review panel determines that the decision to use neither trial shunt was clearly not influenced by treatment assignment (eg, subject dies before surgery), then that subject will be excluded from all analyses. Primary outcome data will be collected for these subjects so that the impact of the exclusions on primary analysis results can be assessed.

Secondary analyses will compare treatment groups as follows: (1) with covariate-adjusted analysis, (2) according to which shunt was actually in place at the conclusion of the operative procedure, and (3) after exclusion of any randomly assigned subjects subsequently found to have been ineligible for the trial at the time of enrollment.

Treatment assignment by prespecified subgroup interaction analyses are planned to estimate the effects of birth weight, preoperative tricuspid regurgitation, type of cerebral perfusion during stage I palliation, experience of the surgeon, and center volume (Table 4).

To monitor the trial for large treatment differences, three formal interim analyses are planned, timed to occur when one quarter, one half, and three quarters of subjects would be expected to reach the 12-month visit. An O'Brien-Fleming stopping boundary with a Lan-DeMets adjustment will be used for this purpose. Adverse event rates by trial arm, as well as death and transplantation rates in aggregate, are reported to the Data and Safety Monitoring Board twice a year. To preserve the type I error rate for primary analysis, death and transplantation rates by treatment arm are reported during prespecified interim analyses only.

This trial is powered to detect differences in the primary composite end point (death or cardiac transplant) by 12 months after random assignment. A review of previous data from the participating centers for infants born in 2002 and 2003 was performed to calculate an estimate of the incidence of death or cardiac transplant for each shunt under consideration. For subjects undergoing a Norwood procedure with MBTS at least 1 year earlier, the incidence of death or transplant by 12 months after surgery was $28 \%$ (65/236). The expected mortality for the RV-PA shunt was based on the three member centers routinely performing the RV-PA shunt exclusively or in a significant proportion of their Norwood procedures. The data on Norwood procedures with RV-PA shunts were sparse, and not all patients had reached 1 year old. Because previously reported data indicate that events after the stage II procedure are rare, ${ }^{11,19}$ all deaths and cardiac transplants, regardless of whether the patient had reached 1 year of age, were counted. For subjects receiving a RV-PA shunt at the three 


\section{TABLE 3. Secondary outcome variables}

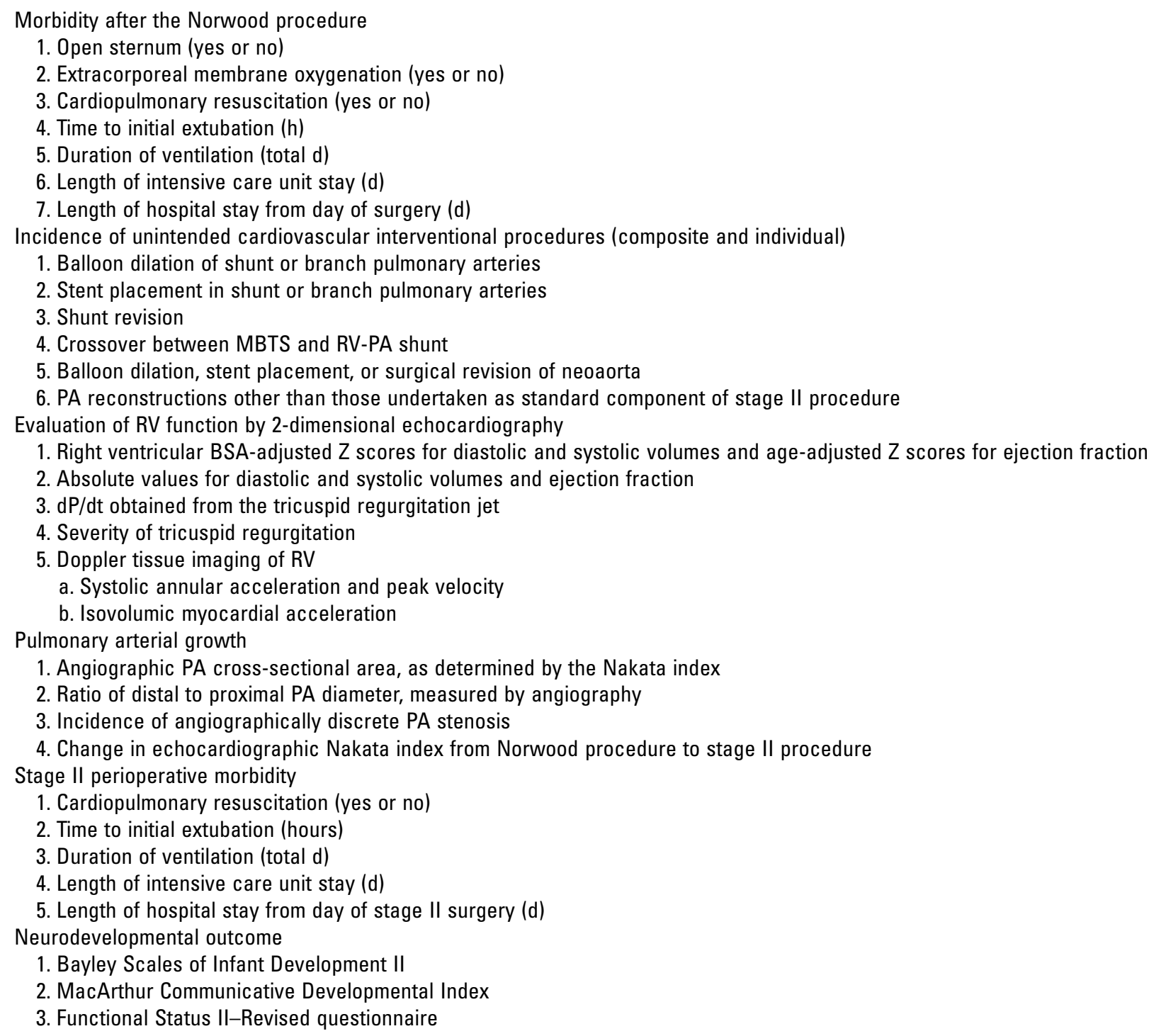

$M B T S$, Modified Blalock-Taussig shunt; $R V$, right ventricle; $P A$, pulmonary artery, $B S A$, body surface area, $d P / d t$, estimated maximum first derivative of ventricular pressure.

centers of interest, the 12-month mortality or transplant rate was assumed to be $16 \%$ (14/87). The trial was thus designed to detect a $12 \%$ difference with $85 \%$ power at a 2 -sided significance level of .05 . After $2 \%$ inflation to account for three interim analyses, this required a total of 466 subjects.

\section{Trial Organization}

The PHN Single Ventricle Reconstruction Study Committee and PHN Steering Committee, together with the National Heart, Lung, and Blood Institute, are responsible for all aspects of this study. The protocol has been approved by an independent Protocol Review Committee and Data and Safety Monitoring Board, and by the institutional review boards at each clinical center and at the Data Coordinating Center. All centers follow the same protocol and study procedures.

\section{Comment}

\section{Rationale for Outcome Measures}

Infants born with HLHS and related conditions have relatively high mortality, unlike neonates with other forms of congenital heart disease. The most fundamental research question to answer in a surgical trial in this population, therefore, is whether the child survives to at least 1 year old, and whether the heart requires replacement before then. The primary outcome, death or cardiac transplant by 12 months after random assignment, reflects this clinical imperative. In addition, this definitive end point should facilitate clear interpretation of the trial results by the medical teams taking care of these patients.

The surgical strategy could affect the postoperative course after the stage I procedure, as well as perioperative morbidity 


\section{TABLE 4. Subgroup factors for estimation of differential treatment effect}

- Birth weight $<2500 \mathrm{~g}$ vs $\leq 2500 \mathrm{~g}$

- Preoperative tricuspid regurgitation proximal jet width $<2.5 \mathrm{~mm}$ vs $\leq 2.5 \mathrm{~mm}$ determined by echocardiography

- Cerebral perfusion during stage I palliation as deep hypothermic circulatory arrest vs regional cerebral perfusion (if subject received both types of cerebral perfusion during surgery, classification was deep hypothermic circulatory arrest)

- Experience of the surgeon as average number of Norwood procedures performed on randomized subjects per year (as a continuous variable) and classified as $\leq 5,5-10,11-15$, or $>15$ procedures/y

- Center volume as average number of Norwood procedures performed on randomized subjects per year (as continuous variable) and classified as $\leq 10,11-25,26-40$, or $>40$ procedures/y

associated with the stage II procedure. Several standard intensive care unit measures were selected as a basis for comparing morbidity, because decreasing intensive care unit morbidity can improve short- and long-term outcomes and reduce health care costs. ${ }^{20,21}$

The need for additional procedures implies incomplete resolution of adverse hemodynamics, carries the risk of further morbidity, and may also affect long-term outcome and health care costs. Several key procedures often used in this population and that might plausibly differ in frequency between the trial arms were selected, including crossover between MBTS and RV-PA shunt, shunt revision, extracorporeal membrane oxygenation, diaphragm plication, and procedures to address stenosis of the shunt, branch pulmonary arteries, or neoaorta.

Systemic (right) ventricular function can theoretically be compromised by either shunt. Although myocardial oxygen delivery and RV function may be optimized when an RVPA shunt is used, RV function has not been systematically evaluated, and the long term effects of the RV ventriculotomy are unknown. Determining whether the type of shunt placed at the time of the Norwood procedure affects later RV function is a crucial component of determining the best surgical management of infants with HLHS. Establishing baseline evaluations and standardizing echocardiographic methods of evaluating RV function will provide early data on a cohort of subjects who can be followed up serially through and beyond Fontan palliation to determine whether a difference in RV function occurs and persists long term, and whether it eventually contributes to failure of staged reconstruction.

The MBTS and RV-PA shunt strategies differ most significantly in their approach to providing pulmonary arterial blood flow, which may influence subsequent PA size, rate of growth, and stenosis. These variables are of clinical significance in their own right, but they also can affect subsequent outcome, because small PA size is a known risk factor for patients undergoing both the stage II palliation and the Fontan procedure. $^{22-24}$ The impact of the MBTS relative to the RVPA shunt on PA growth and stenosis will be evaluated.

Multiple studies have demonstrated that preschool and school-aged children with HLHS have neurocognitive and behavioral abnormalities. Risk factors for such abnormalities include underlying congenital structural central nervous system abnormalities, genetic syndromes, disturbed fetal cerebral hemodynamics, preoperative seizures, intraoperative events related to $\mathrm{CPB}$, and such postoperative factors as low cardiac output syndrome, seizures, and longer of hospital stay. $^{25-29}$

It is hypothesized that relative to the MBTS the RV-PA shunt may reduce neurodevelopmental impairment through improved postoperative oxygen delivery, less steal from the systemic circulation through the shunt into the lungs, and higher diastolic blood pressure. Measures of neurodevelopmental status will profile the motor and cognitive capabilities of study subjects. The effects of known modifiers of neurodevelopment will be evaluated in the analysis. One such modifier is the $A P O E^{*} E 2$ allele, recently found to be associated with worse neurologic outcome in patients undergoing CPB. ${ }^{30}$ APOE genotype will be assessed as a component of neurodevelopmental outcome.

\section{Limitations}

Blinding of subjects and caregivers is desirable in clinical trials because of the potential for bias in medical care and in adjudication of treatment outcomes. Practical considerations preclude blinding in most surgical trials, however, including this one. In the absence of blinding, it is important to ensure that random assignment is conducted properly and that end points are assessed objectively. The PHN's standard quality assurance process will be used for close monitoring of screening and randomization. Although the primary end point is a composite, most of the events are expected to be deaths, an objective measure. If a significant number of cardiac transplants occur, the transplant criteria will be adjudicated independently in each case.

This trial, like many other studies of rare conditions, is powered to detect a relatively large difference in the primary end point (16\% vs $28 \%$ ), so a smaller but clinically important difference might not be detected. Powering the study to detect a smaller difference, however, would require many more subjects and thus possibly a longer study duration. The decision to adopt the current design was made to avoid the loss of equipoise and potential confounding changes in 
surgical techniques and perioperative care that could occur over a longer time.

To maximize enrollment, the number of secondary outcome measures is intentionally limited to minimize the number of extra measurements and thus the burden on subjects and families. For example, although 48-hour electroencephalographic monitoring or head magnetic resonance imaging might provide mechanistic data to explain abnormalities in neurodevelopment, these studies are not included. In addition, the trial may be underpowered for some of the secondary end point analyses.

\section{Importance of Knowledge to Be Gained}

Techniques for management of congenital heart lesions have been adopted solely on the basis of expert consensus and retrospective analysis, rather than on data from prospective, randomized clinical trials. The primary goal of the PHN is to promote evidence-based clinical care for children and adults with congenital heart disease. This trial offers a unique opportunity for systematic comparison of two surgical procedures for which equipoise exists among surgeons and for determination of the time course of risk and benefit for each procedure. Not only will the main results provide an unbiased estimate of relative efficacies of the two shunts, but standardized information characterizing the clinical course in a large unselected cohort of infants with HLHS will be obtained for the first time. The results of this study will make an important contribution to the management of the highest-risk group of patients with congenital heart disease. Another important contribution of this trial will be establishment of the basis for long-term followup of a well-characterized cohort of patients with little systematic information currently available. Finally, this trial provides a feasible model of collaborative research for the evaluation of other operative interventions in patients with congenital cardiovascular malformations.

\section{References}

1. Alsoufi B, Bennetts J, Verma S, Caldarone CA. New developments in the treatment of hypoplastic left heart syndrome. Pediatrics. 2007; 119:109-17.

2. Sano S, Ishino K, Kawada M, Arai S, Kasahara S, Asai T, et al. Right ventricle-pulmonary artery shunt in first-stage palliation of hypoplastic left heart syndrome. J Thorac Cardiovasc Surg. 2003; 126:504-10.

3. Ohye RG, Ludomirsky A, Devaney EJ, Bove EL. Comparison of right ventricle to pulmonary artery conduit and modified Blalock-Taussig shunt hemodynamics after the Norwood operation. Ann Thorac Surg. 2004;78:1090-3.

4. Mahle WT, Cuadrado AR, Tam VK. Early experience with a modified Norwood procedure using right ventricle to pulmonary artery conduit. Ann Thorac Surg. 2003;76:1084-9.

5. Lai L, Laussen PC, Cua CL, Wessel DL, Costello JM, del Nido PJ, et al. Outcomes after bidirectional Glenn operation: Blalock-Taussig shunt versus right ventricle-to-pulmonary artery conduit. Ann Thorac Surg. 2007;83:1768-73
6. Pizarro C, Norwood WI. Right ventricle to pulmonary artery conduit has a favorable impact on postoperative physiology after Stage 1 Norwood: preliminary results. Eur J Cardiothorac Surg. 2003;23:991-5.

7. Azakie A, Martinez D, Sapru A, Fineman J, Teitel D, Karl TR. Impact of right ventricle to pulmonary artery conduit on outcome of the modified Norwood procedure. Ann Thorac Surg. 2004;77:1727-33.

8. Tanoue Y, Kado H, Shiokawa Y, Fusazaki N, Ishikawa S. Midterm ventricular performance after Norwood procedure with right ventricularpulmonary artery conduit. Ann Thorac Surg. 2004;78:1965-71.

9. Cua CL, Thiagarajan RR, Gauvreau K, Lai L, Costello JM, Wessel DL, et al. Early postoperative outcomes in a series of infants with hypoplastic left heart syndrome undergoing stage 1 palliation operation with either modified Blalock-Taussig shunt or right ventricle to pulmonary artery conduit. Pediatr Crit Care Med. 2006;7:238-44.

10. Kussman BD, Gauvreau K, DiNardo JA, Newburger JW, Mackie AS, Booth KL, et al. Cerebral perfusion and oxygenation after the Norwood procedure: comparison of right ventricle-pulmonary artery conduit with modified Blalock-Taussig shunt. J Thorac Cardiovasc Surg. 2007;133: 648-55.

11. Pizarro C, Malec E, Maher KO, Januszewska K, Gidding SS, Murdison KA, et al. Right ventricle to pulmonary artery conduit improves outcome after Stage 1 Norwood for hypoplastic left heart syndrome. Circulation. 2003;108(Suppl 1):II155-60.

12. Mair R, Tulzer G, Sames E, Gitter R, Lechner E, Steiner J, et al. Right ventricular to pulmonary artery conduit instead of modified BlalockTaussig shunt improves postoperative hemodynamics in newborns after the Norwood operation. J Thoracic Cardiovasc Surg. 2003;126:1378-84.

13. Tabbutt S, Dominguez TE, Ravishankar C, Marino BS, Gruber PJ, Wernovsky G, et al. Outcomes after the stage 1 reconstruction comparing the right ventricular to pulmonary artery conduit with the modified Blalock Taussig shunt. Ann Thorac Surg. 2005;80:1582-91.

14. Mahony L, Sleeper LA, Anderson PA, Gersony WM, McCrindle BW, Minich LL, et al. The Pediatric Heart Network: a primer for the conduct of multicenter studies in children with congenital and acquired heart disease. Pediatr Cardiol. 2006;27:191-8.

15. Bando K, Turrentine MW, Sun K, Sharp TG, Caldwell RL, Darragh RK, et al. Surgical management of hypoplastic left heart syndrome. Ann Thorac Surg. 1996;62:70-7.

16. Bove EL. Current status of staged reconstruction for hypoplastic left heart syndrome. Pediatr Cardiol. 1998;19:308-15.

17. Breymann T, Kirchner G, Blanz U, Cherlet E, Knobl H, Meyer H, et al. Results after Norwood procedure and subsequent cavopulmonary anastomoses for typical hypoplastic left heart syndrome and similar complex cardiovascular malformations. Eur J Cardiothorac Surg. 1999;16: 117-24.

18. Jenkins PC, Flanagan MF, Jenkins KJ, Sargent JD, Canter CE, Chinnock RE, et al. Survival analysis and risk factors for mortality in transplantation and staged surgery for hypoplastic left heart syndrome. J Am Coll Cardiol. 2000;36:1178-85.

19. Iannettoni MD, Bove EL, Mosca RS, Lupinetti FM, Dorostkar PC, Ludomirsky A, et al. Improving results with first-stage palliation for hypoplastic left heart syndrome. J Thoracic Cardiovascular Surg. 1994; 107:934-40.

20. Limperopoulos C, Majnemer A, Shevell MI, Rohlicek C, Rosenblatt B, Tchervenkov C, et al. Predictors of developmental disabilities after open heart surgery in young children with congenital heart defects. J Pediatr. 2002;141:51-8.

21. Newburger JW, Wypij D, Bellinger DC, du Plessis AJ, Kuban KC, Rappaport LA, et al. Length of stay after infant heart surgery is related to cognitive outcome at age 8 years. J Pediatr. 2003;143:67-73.

22. Douglas WI, Goldberg CS, Mosca RS, Law IH, Bove EL. Hemi-Fontan procedure for hypoplastic left heart syndrome: outcome and suitability for Fontan. Ann Thorac Surg. 1999;68:1361-8.

23. Mosca RS, Kulik TJ, Goldberg CS, Vermilion RP, Charpie JR, Crowley DC, et al. Early results of the Fontan procedure in one hundred consecutive patients with hypoplastic left heart syndrome. $J$ Thorac Cardiovasc Surg. 2000;119:1110-8.

24. Valera Martinez FJ, Caffarena Calvar J, Gomez-Ullate JM, GomezPlana Usero J, Carrasco JI, Saez JM, et al. [Risk factors in the Glenn bidirectional shunt as an intermediate procedure before Fontan correction] [Spanish]. Rev Esp Cardiol. 1999;52:903-9. 
25. Wernovsky G, Stiles KM, Gauvreau K, Gentles TL, duPlessis AJ, Bellinger DC, et al. Cognitive development after the Fontan operation. Circulation. 2000;102:883-9.

26. Mahle WT, Clancy RR, Moss EM, Gerdes M, Jobes DR, Wernovsky G. Neurodevelopmental outcome and lifestyle assessment in school-aged and adolescent children with hypoplastic left heart syndrome. Pediatrics. 2000;105:1082-9.

27. Goldberg CS, Schwartz EM, Brunberg JA, Mosca RS, Bove EL, Schork MA, et al. Neurodevelopmental outcome of patients after the Fontan operation: A comparison between children with hypoplastic left heart syndrome and other functional single ventricle lesions. $J$ Pediatr. 2000;137:646-52.

28. Hoffman GM, Mussatto KA, Brosig CL, Ghanayem NS, Musa N, Fedderly RT, et al. Systemic venous oxygen saturation after the Norwood procedure and childhood neurodevelopmental outcome. J Thorac Cardiovasc Surg. 2005;130:1094-100.

29. Mahle WT, Visconti KJ, Freier MC, Kanne SM, Hamilton WG, Sharkey AM, et al. Relationship of surgical approach to neurodevelopmental outcomes in hypoplastic left heart syndrome. Pediatrics. 2006; 117:e90-7.

30. Gaynor JW, Gerdes M, Zackai EH, Bernbaum J, Wernovsky G, Clancy RR, et al. Apolipoprotein E genotype and neurodevelopmental sequelae of infant cardiac surgery. J Thorac Cardiovasc Surg. 2003; 126:1736-45.

\section{Appendix: Pediatric Heart Network Investigators}

National Heart, Lung, and Blood Institute. Gail Pearson (Project Director), Victoria Pemberton (Deputy Project Director), Rae-Ellen Kavey, Judith Massicot-Fisher, Marsha Mathis, Mario Stylianou

Network Chair. University of Texas Southwestern Medical Center, Lynn Mahony

Data Coordinating Center. New England Research Institutes, Lynn Sleeper (PI), Steven D. Colan, Patty Connell, Victoria Murtov, Lisa Wruck, Lisa Virzi, David F. Teitel

Core clinical site investigators. Children's Hospital Boston, Jane Newburger (PI), Peter Laussen, Pedro del Nido, Roger Breitbart, Jami Levine, Ellen McGrath, Carolyn DunbarMasterson; Children's Hospital of New York, Daphne Hsu (PI), Ashwin Prakash, Ralph Mosca, Darlene Servedio; Children's Hospital of Philadelphia, Victoria L. Vetter (PI), Sarah Tabbutt, J. William Gaynor (Study Co-Chair), Marisa Nolan,
Stephanie Piacentino; Cincinnati Children's Medical Center, D. Woodrow Benson (PI), Catherine Dent, Lois Bogenschutz, Teresa Barnard; Duke University, Page A. W. Anderson (PI), Piers Barker, James Jaggers, Charlie Sang, Jr, Wesley Covitz, Theodore Koutlas, Ming Xu, Lori Jo Sutton, Kari Crawford; Medical University of South Carolina, J. Philip Saul (PI), Andrew Atz, Scott Bradley, Eric Graham, Girish Shirali, Teresa Atz; Primary Children's Medical Center, Salt Lake City, Utah, LuAnn Minich (PI), Michael Pulchalski, John Hawkins, Ruth Mestas, Marian Sharrow; Hospital for Sick Children, Toronto, Brian McCrindle (PI) Joel Kirsh, Chris Caldarone, Elizabeth Radojewski, Susan McIntyre; University of Michigan, Richard G. Ohye (Study Chair), Caren S. Goldberg, Cheryl Nowak; Children's Hospital of Wisconsin, Nancy Ghanayem, James Tweddell, Kathy Mussatto, Michele Frommelt, Lisa Young-Borkowski

Auxiliary sites. Children's Hospital Los Angeles, Alan Lewis, Vaughn Starnes, Nancy Pike; Congenital Heart Institute of Florida, Jeff Jacobs, James, Huhta, Tina Merola; Emory University, Kirk Kanter, William Mahle, Joel Bond; Nemours Cardiac Center, Christian Pizarro, Carol Prospero; University of Texas Southwestern Medical Center, Tia Tortoriello, Deborah McElroy, Deborah Town.

Angiography core laboratory. Duke University, John Rhodes

Echocardiography core laboratories. Children's Hospital of Wisconsin, Peter Frommelt; Children's Hospital Boston, Gerald Marx.

Genetics core laboratory. Children's Hospital Philadelphia, Catherine Stolle

Protocol Review Committee. Michael Artman (Committee Chair), Erle Austin, Timothy Feltes, Julie Johnson, Thomas Klitzner, Jeffrey Krischer, G. Paul Matherne

Data and Safety Monitoring Board. John Kugler (Board Chair), David J. Driscoll, Mark Galantowicz, Sally A. Hunsberger, Thomas J. Knight, Holly Taylor, Catherine L. Webb 